\title{
LA FORMULACIÓN DE LOS OBJETIVOS EN ARTÍCULOS DE INVESTIGACIÓN CIENTÍFICA EN CUATRO DISCIPLINAS: HISTORIA, LINGÜÍSTICA, LITERATURA Y BIOLOGÍA*
}

Cristian González Arias**

Resumen: Analizar las propiedades estructurales y funcionales de los productos textuales de la actividad cientifica ayuda a comprender el rol del lenguaje en la construcción y difusión del conocimiento. En el presente articulo se analiza la formulación de los objetivos en artículos de investigación científica (AIC) en cuatro disciplinas: Historia, Lingüistica, Literatura y Biología. El propósito fue identificar patrones de formulación de los objetivos, los apartados donde son presentados y si existen diferencias entre las disciplinas estudiadas. Se analizaron 40 articulos, diez de cada una de las disciplinas. Como resultado, identificamos cinco patrones en la formulación de objetivos, los que son presentados indistintamente en el resumen $y$ en la introducción. Sin embargo, se aprecian diferencias notables entre los AIC de las disciplinas estudiadas. Asimismo, con frecuencia se produce una ambigüedad entre presentar el objetivo de la investigación y el objetivo del articulo, resaltando el carácter metonímico y realizativo del AIC.

Palabras clave: Discurso especializado. Articulo de investigación. Objetivos de investigación.

\section{INTRODUCCIÓN}

Comunicar los resultados de una investigación es una etapa fundamental en el proceso de producción de conocimientos. Podemos afirmar que toda investigación se completa con la publicación de un documento que sintetiza los resultados, poniendo en perspectiva los hallazgos y conclusiones. Estas publicaciones se pueden materializar en un libro, en un artículo o en otro tipo de informe de investigación, como tesis de grado o comunicaciones en congresos.

En el contexto de los estudios del discurso especializado y de la enseñanza de la escritura en las disciplinas, se han caracterizado los géneros discursivos que se usan en la comunicación científica en diversas disciplinas y se han descrito desde diferentes perspectivas (PARODI, 2007). Particularmente, se ha manifestado un interés por describir la

\footnotetext{
* Proyecto Fondecyt 1090030.

** Profesor Asociado, Pontificia Universidad Católica de Valparaíso. Doctor en Lingüística. Email: cristian.gonzalez@ucv.cl
} 
estructura retórica de textos en el ámbito académico y científico (RENKEMA, 1999; HYLAND, 2002; SABAJ; TORO; FUENTES, 2011; PARODI, 2010; IBÁÑEZ, 2010).

En el caso de los artículos de investigación científica (AIC), la estructura y función de su organización textual se ha ido estandarizando en el tiempo de acuerdo a las tradiciones y estilos de cada disciplina. En este proceso de estandarización han contribuido fuertemente la especialización de las revistas científicas, los procesos de evaluación de pares y, más recientemente, la masiva incorporación de las revistas en sistemas de acreditación e indexación, los que han establecido una serie de normas y procedimientos para aceptar un artículo para su publicación (SABAJ; MATSUDA; FUENTES, 2010).

Debido a la importancia que ha adquirido la publicación de AIC, se han editado una serie de manuales y guías para la escritura de este tipo de géneros (MARTINSSON, 1983; CAIVANO, 1995; DAY, 2005; VALDERRAMA, 2005; SABAJ, 2007). En todos estos trabajos, existe consenso en que se escribe un AIC para informar los resultados de una investigación empírica o teórica que busca la generación de conocimiento nuevo. A su vez, en toda investigación los objetivos científicos son elementos clave, por lo que se espera que sean presentados en el AIC y que estos ocupen un lugar preponderante en la organización retórica del texto. A pesar de la importancia de la formulación de los objetivos en la escritura de un AIC, existen escasas descripciones empíricas de la forma específica en que éstos se concretizan textualmente. Adoptando la perspectiva Semiolingüística del Análisis del Discurso y en el marco del Análisis Retórico de Géneros, el propósito de esta investigación fue identificar patrones de formulación de los objetivos, los apartados dónde son presentados y si existen diferencias entre los AIC de las disciplinas estudiadas.

El artículo está organizado de la siguiente manera: revisamos primero el rol de los objetivos científicos en el marco de una investigación y de su publicación en un AIC. Seguidamente, realizamos una discusión respecto de los trabajos que han descrito las características retóricas en AIC, específicamente, de la sección Resumen e Introducción. En la segunda parte de este documento, se presenta la investigación, los resultados y las conclusiones. 


\section{LOS OBJETIVOS CIENTÍFICOS EN METODOLOGÍA DE LA INVESTIGACIÓN}

De la revisión de algunos manuales que se ocupan en general de la descripción de los pasos y elementos centrales de una investigación, encontramos algunas referencias comunes que dan cuenta de la posición preponderante que tienen los objetivos científicos en una investigación. Se plantea que los objetivos de investigación son centrales en el proceso de investigación, pues estos surgen de establecer "qué pretende la investigación...son las guías del estudio y durante todo su desarrollo deben tenerse presente" (HERNÁNDEZ, FERNÁNDEZ; BAPTISTA, 2004, p. 11).

Vale considerar el estatus privilegiado de los objetivos en relación a otros elementos que organizan el proceso investigativo también centrales como las preguntas y las hipótesis en una investigación: "Las hipótesis sustituyen a los objetivos y preguntas de investigación para guiar el estudio" (HERNÁNDEZ et al., 2004, p. 78).

También se puede establecer que los objetivos son la meta de una investigación (ARIAS, 1999). Para Guanipa (2008, p. 2) "los objetivos de investigación son las metas, propósitos o fines trazados por el investigador en concordancia con los aspectos que desea verificar y descubrir". Por su parte, Flórez y Tobón (2003), los objetivos están directamente relacionados con los tipos de conocimientos que se pretenden alcanzar en relación con las preguntas que constituyen el problema de investigación. En este contexto, no cabe duda que los objetivos de investigación guían el proceso de investigación y se constituyen en un elemento central de ese proceso; por lo tanto, se espera que cualquier reporte de una investigación considere los objetivos de investigación al momento de publicar los resultados, anunciados como objetivos, propiamente tal, pero también como propósitos, metas o fines de la investigación reportada. Igualmente, vale destacar que, desde un punto de vista retórico, la claridad de los objetivos y la presencia de la adecuada ubicación en un artículo también permitirían focalizar la atención del lector, facilitándole la comprensión de dicho artículo. 


\section{EL ARTÍCULO DE INVESTIGACIÓN COMO GÉNERO DISCURSIVO}

En los últimos años, numerosos trabajos se han abocado a la descripción de géneros del discurso especializado (BECHER, 1987; BAZERMAN, 1988; PARODI, 2007; PARODI; IBÁÑEZ; VENEGAS; GUTIÉRREZ, 2008; SABAJ; MATSUDA; FUENTES, 2010). Según Parodi, Ibáñez, Venegas y Gutiérrez (2008, p. 53) un AIC es un "género discursivo cuyo macropropósito comunicativo es persuadir respecto de un determinado punto de vista, asumido en una revisión teórica o respecto de los resultados obtenidos de un estudio empírico".

Se agrega, además, que se trata de un tipo de texto altamente especializado dado que el escritor y el lector son expertos en un tema. Igualmente, se propone que debe presentarse información suficiente a fin de que los posibles usuarios de los datos puedan: a) evaluar las observaciones, b) repetir los experimentos, y c) evaluar los procesos intelectuales (Day, 2005).

A partir del modelo CARS, desarrollado por Swales (1990, 2004), se han realizado descripciones detalladas de la estructura retórica de los textos del género AIC. Puesto que el modelo fue originalmente creado para explicar la estructura de las introducciones, es justamente esta sección, junto con el resumen, la que ha recibido mayor atención, tal como lo evidencian los trabajos de Martín (2003), Lorés (2004), Samraj (2005), Rimrott (2007) y Dzung (2008), que se centran en el resumen; y las pesquisas de Grookes (1986), Falahati (1999), Samraj (2002, 2005), Fakhri (2004), Ferrari (2006) y Ozturk (2007), que se abocan al estudio de las introducciones.

Una de las observaciones más comunes es la descripción del AIC con la estructura IMRD (SWALES, 1990) que resume el AIC en cuatro partes principales: Introducción, Métodos, Resultados y Discusión. Como señala Day (2005, p. 11): "En las ciencias básicas, la forma más corriente de designar esas partes componentes es: Introducción, Métodos, Resultados y Discusión". El mismo autor agrega que el orden básico IMRD es tan eminentemente lógico que, cada vez más, se está utilizando para muchas otras clases de textos de carácter expositivo (DAY, 2005). 
Específicamente en torno al apartado donde se deben escribir los objetivos, se ha señalado que estos deberían aparecer en el resumen y en la introducción. Por su parte, Astete (2001) propone unas pautas para la lectura crítica de AIC, señalando algunas características cualitativas de los artículos de investigación científica en el área de la salud. Establece una relación entre el processo investigativo y sus correspondencias con la estrutura del AIC, destacando las cualidades de cada uno de los segmentos. En este ejercicio, sitúa los objetivos en la etapa conceptual que corresponde a la introducción.

\subsection{LA ORGANIZACIÓN RETÓRICA DEL RESUMEN}

Respecto del resumen, se ha planteado que este debería permitir al lector, que está buscando información especializada respecto de un tema específico, decidir si el artículo que es resumido es o no pertinente para los intereses de un investigador. Al respecto se señala: “...el resumen debe incluir, de manera detallada pero sucinta, los resultados y conclusión del artículo, ajustándose al espacio a ello destinado, e indicar, dentro de estos límites, todo tipo de información nueva que contenga el artículo" (MARTINSSON, 1983, p. 9). Igualmente, Venegas (2007, p. 80) señala:

Desde el punto de vista semántico el resumen corresponde a una globalización (condensación de la información en unidades menores) y a una conceptualización de la red de contenidos del texto. En este sentido, proponemos denominar a este proceso de globalización como 'macrosemantización', ya que el resumen es la textualización de un significado que representa de modo abstracto el significado total del contenido del artículo.

Asimismo, Day (2005) señala que el resumen puede considerarse como una versión en miniatura del artículo. Debe ofrecer un sumario breve de cada una de las secciones principales. El mismo autor precisa que el contenido del resumen tendría que: a) indicar los objetivos principales y el alcance de la investigación, b) describir los métodos empleados, c) resumir los resultados, y d) enunciar las conclusiones 
principales. Igualmente, se propone que el resumen debería escribirse en pretérito, precisamente porque refiere a un trabajo ya realizado, así como, se advierte que el resumen no debería presentar ninguna información ni conclusión que no esté en el artículo (DAY, 2005).

Además, se ha señalado que el resumen tiene la misma estructura lógica que el contenido del informe de investigación, comúnmente su estructura retórica más común se conoce por las siglas IMR: Introducción, Método y Resultados" (VENEGAS, 2005; MOYANO, 2000). De igual manera, Swales (2004) y Swales y Feak (2010) han descrito la estructura del resumen en base a la noción de movida retórica, la que se define como un segmento textual que cumple una determinada función en la organización del material textual. A continuación, se sintetizan las partes principales que debería contener el resumen de acuerdo a los autores mencionados.

Tabla 1: Modelo de organización retórica del apartado resumen (SWALES; FEAK, 2010, p. 5, traducción nuestra)

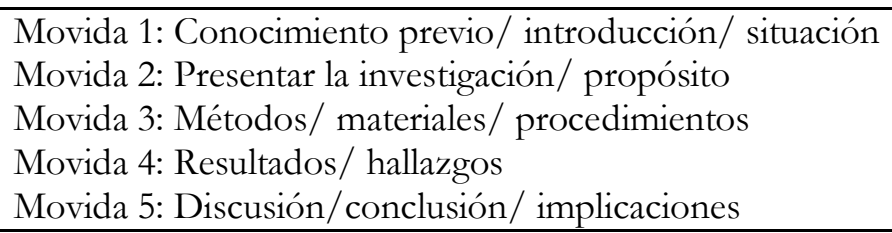

Como podemos apreciar, para estos autores los objetivos considerados de manera general como propósitos son parte de la Movida 2 en la que se presenta la investigación propiamente tal y, naturalmente, su propósito. Se espera, entonces, que en el resumen se describa la investigación en términos generales y de manera esquemática, para lo cual los objetivos de la investigación deberían ocupar un lugar preponderante.

Corresponde hacer aquí una breve reflexión frente a la relación entre el resumen y el artículo en su totalidad. Dado que el resumen es una parte del artículo, posee una relación especial con las otras partes (como la introducción o las conclusiones), ya que sintetiza la información que contiene el artículo en su totalidad. En tales términos, el resumen no debería incluir información que no haya sido abordada en el artículo en su totalidad. Esto se desprende naturalmente por el concepto de 
resumen, por lo tanto, sería un error que el resumen incluyera información relevante como objetivo o metodología que no sea también mencionada en el resto del AIC.

\subsection{LA ORGANIZACIÓN RETÓRICA DE LA INTRODUCCIÓN}

Otro segmento estudiado con cierta acuciosidad ha sido el apartado introducción. Se define la introducción como una pieza textual, relativamente compleja, que debe cumplir variadas funciones. Sin embargo, se podría sintetizar que la función principal de la introducción es plantear la finalidad del artículo (MARTINSSON, 1983). Asimismo, Venegas (2005, p. 15) plantea que dentro de las funciones que la introducción debe cumplir se encuentran la de "proponer un objetivo para enfrentar el problema de investigación". Para Day (2005, p. 32) la introducción tiene como finalidad:

Suministrar suficientes antecedentes para que el lector pueda comprender y evaluar los resultados del estudio sin necesidad de consultar publicaciones anteriores sobre el tema. Debe presentar también el fundamento racional del estudio. Por encima de todo, hay que manifestar breve y claramente cuál es el propósito al escribir el artículo.

Vale destacar aquí que se presenta la idea de propósito asociada a la organización retórica del escrito, lo que podríamos identificar como objetivo retórico del artículo propiamente tal, el que se confunde con el objetivo de la investigación. La precisión anterior nos introduce en la discusión en torno a la relación entre la actividad de investigar y el reporte de la investigación. Tal como lo han descrito los clásicos trabajos de Knorr-Cetina (1981) y de Latour y Woolgar (1986), se establece que el proceso de realizar una investigación es inverso al de reportarla en la forma de un escrito. No obstante, podemos destacar el carácter mimético del artículo respecto de la investigación y, lo que ocurre con frecuencia, el carácter metonímico del artículo respecto de la investigación, en el sentido de que si bien el AIC informa una 
investigación realizada, la comunicación de los resultados se constituye como una parte del proceso global de investigar.

Así como fue descrito para el apartado resumen, Swales (1990, 2004) también hace una descripción de las movidas retóricas principales del apartado introducción de un AIC. En este esquema elaborado en 1990 y reformulado en 2004, como se puede ver en la Tabla 2, se asocia la formulación de objetivos de investigación en lo que denominan la Movida 3 de la introducción. En el esquema de 1990 se señala que correspondería a "ocupar el nicho", naturalmente, como respuesta a la previa identificación de la necesidad, no de escribir el artículo, sino de realizar la investigación. En el esquema revisado el año 2004, se nomina a la movida "presentar el trabajo", probablemente, como una forma de ampliar la denominación anterior y, con ello, recoger las diferentes formas como se escriben las introducciones.

Tabla 2: Las movidas retóricas en el apartado introducción

\begin{tabular}{|l|l|}
\hline Modelo CARS, Swales (1990) & Modelo CARS revisado, Swales (2004) \\
\hline Movida 1: Establecer el territorio & Movida 1: Establecer el territorio \\
Movida 2: Establecer el nicho & Movida 2: Establecer el nicho \\
Movida 3: Ocupar del nicho & Movida 3: Presentar el trabajo \\
\hline
\end{tabular}

Por su parte, Moyano (2000) señala que la introducción debe incluir: a) definición del problema, b) antecedentes, c) justificación, d) objetivos y e) limitaciones y alcances del trabajo. Desde el ámbito de la enfermería, Henríquez y Zepeda (2004) hacen sugerencias de cómo escribir un AIC y plantean explícitamente que se deberían presentar los objetivos de investigación tanto en el Resumen como en la introducción. Junto a ellos, Morales, González y Tona (2006) realizan una investigación sobre 50 artículos del área de la odontología y plantean que solo el 30\% de las introducciones presentaban antecedentes del problema investigado y de los objetivos de la investigación. Por otra parte, basado en un estudio de 40 artículos en el área de medicina, Morales (2008) señala que el 98\% incluyó en la introducción el objetivo de la investigación (aunque no hace referencia a los resúmenes de dichos artículos).

A partir de los antecedentes recogidos, podemos sintetizar algunas de las ideas centrales respecto de lo que se ha descrito en torno al reporte de los objetivos de la investigación que motivan la publicación de un 
AIC. En términos generales, tenemos que señalar que se espera que en un AIC se planteen los objetivos de la investigación en el resumen y en la introducción, de manera que el lector pueda ser orientado respecto de la lectura. La mención de los objetivos en el resumen orientaría al lector para seguir leyendo el resto del artículo o, probablemente, adquirir determinado artículo en el caso de que no esté disponible gratuitamente. A partir de los antecedentes recogidos, no se descarta que se puedan presentar los objetivos en otro apartado.

También es importante destacar que los objetivos podrían ser formulados como metas, propósitos, fines e, incluso, como la intención del autor en las actividades de investigación realizadas. Es decir, no solo podrían ser explicitados con el nombre de "objetivos", sino con palabras equivalentes a metas, fines o intenciones.

\section{LA INVESTIGACIÓN}

La investigación tuvo un carácter descriptivo y se realizó mediante procedimientos cualitativos y conteo de frecuencia. Se recolectó un corpus de 40 artículos, 10 de cada una de las disciplinas estudiadas: Historia, Lingüística, Literatura y Biología. Todos los artículos fueron seleccionados aleatoriamente de la sección artículos de revistas científicas del indexador Scielo-Chile.

La metodología de análisis consistió en identificar el enunciado o segmento textual en el que se mencionara o hiciera alusión a los objetivos de manera directa o indirecta, lo que permitió que se extrajera, incluso, más de un segmento textual en un solo apartado. En una primera etapa, se consignaron todos los segmentos textuales que indicaran explícitamente los objetivos, propósitos, metas u otros equivalentes, que dieran cuenta, de algún modo, de la actividad científica que motiva la escritura del artículo. En una segunda etapa se consideraron otras formas indirectas o implícitas de indicar el objetivo o propósito señalando la actividad global que aparecía como finalidad o meta. Cada una de estas menciones se consideró como una ocurrencia del fenómeno de estudio y se consignó el apartado de donde fueron extraídos, esto es, si aparecieron en el Resumen, Introducción u Otro. 
Posteriormente, se procedió a clasificar los segmentos textuales identificados de acuerdo con la estructura formal del enunciado y atendiendo a si estos enunciados hacían referencia directa a los objetivos de investigación reportada o a los objetivos del artículo que reporta la investigación o a los objetivos del autor del AIC.

El establecimiento de patrones de formulación de objetivos se realizó mediante la elaboración de categorías formales y funcionales que serán descritas en el apartado dedicado a los resultados. El reconocimiento de que de un segmento textual correspondía o no a los objetivos se realizó mediante la colaboración de tres investigadores, lo que permitió desambiguar por consenso las situaciones donde hubo discrepancias y se elaboraron colectivamente los patrones que permitieron clasificar las diversas maneras de incluir los objetivos en un AIC.

\section{RESULTADOS Y DISCUSIÓN}

A continuación se presentarán los resultados obtenidos que corresponden a la descripción de tres aspectos principales: los patrones que se identificaron en la formulación de objetivos en los AIC, el apartado en que se formulan (Resumen, Introducción u Otro) y las diferencias entre los AIC de las distintas disciplinas estudiadas.

\subsection{PATRONES DE FORMULACIÓN DE OBJETIVOS EN EL AIC}

Del análisis de todos los segmentos textuales que directa o indirectamente referían a los objetivos, hemos identificado cinco patrones principales que son presentados a continuación:

Tipo A. Explicita el objetivo de la investigación

Tipo B. Explicita el objetivo del artículo

Tipo C. Explicita el objetivo del autor

Tipo D. Señala lo que hace el artículo

Tipo E. Señala lo que se hace en el documento 
A continuación, presentaremos una descripción y ejemplos de cada uno de estos patrones.

\subsubsection{EXPLICITA EL OBJETIVO DE LA INVESTIGACIÓN}

Del total de segmentos textuales identificados como formulación explícita de objetivos o propósitos en todos los textos en todos los apartados (60 segmentos) solo 11 fueron formulados como presentación (explícita) de objetivos de una investigación realizada con independencia de la actividad de escribir el artículo, esto es el 18,3\%.

Este tipo de formulación se consignó como "Explicita los objetivos de la investigación", la que corresponde a una aseveración delocutiva que sigue un patrón como el que se describe a continuación:

El objetivo de esta investigación + verbo ser en pasado + verbo en infinitivo

Como podemos apreciar en los ejemplos que señalamos a continuación, destaca el verbo en pasado que marca la distancia enunciativa entre la actividad de investigar y la actividad de escritura en la que se reporta la investigación.

Ejemplo 1: "El objetivo de este trabajo fue analizar las propiedades morfológicas y semánticas del sufijo -ero dentro de las creaciones neológicas". LING10.

Ejemplo 2: "Los objetivos principales del presente trabajo fueron: (1) proporcionar información acerca de características genético poblacionales (variabilidad genética, heterogeneidad genética) de los pumas en la citada región de los Andes bolivianos mediante el análisis de siete loci microsatélites hiperpolimórficos a partir de un método no invasivo (heces y fragmentos de pieles de animales ya muertos), y (2) determinar posibles diferencias genéticas entre esta población andina boliviana y los pumas de otros países latinoamericanos (Colombia, Ecuador, Perú, Venezuela y Amazonia brasileña occidental)". BIO3. 
En los ejemplos 1 y 2, se presenta la investigación como el "trabajo" cuyo objetivo se presenta con el verbo ser en pasado "fue" o "fueron", respectivamente. También fue considerada como una forma explícita formulaciones con verbos que indican un propósito o expectativa como en el Ejemplo 3.

Ejemplo 3: "En consecuencia, en este estudio se esperaba observar variaciones concomitantes en las infracomunidades parasitarias". BIO9.

\subsubsection{EXPLICITA EL OBJETIVO DEL ARTÍCULO}

Otra manera de formular los objetivos de la investigación la hemos categorizado como "Explicita el objetivo del artículo". En este caso, se presenta al artículo en una relación metonímica con la investigación, es decir, artículo e investigación se confunden. Esta situación se observó en un $11,7 \%$ de los segmentos identificados. El patrón característico lo hemos descrito como una aserción delocutiva que se estructura de la siguiente manera:

El artículo tiene por objetivo + verbo en infinitivo

El objetivo de este artículo es + verbo en infinitivo

En todas estas ocasiones, la atribución de un objetivo al artículo se formuló en tiempo presente. Vale destacar que no se encontraron bajo este patrón objetivos que pudieran ser atribuidos exclusivamente a la actividad de escribir con independencia de la actividad de investigar.

Ejemplo 4: "Este trabajo tiene por objetivo analizar el poemario La Hybris (2007) de la escritora argentina Alicia Genovese, considerando la configuración del yo lírico y, asimismo, el tipo de política genérico-sexual que éste despliega". LIT2. 
Ejemplo 5: "El propósito de este artículo es realizar un examen crítico general de la noción de género en dos orientaciones contemporáneas de la lingüística, que se distinguen por los aportes tanto teóricos como empíricos realizados a esta temática: la Lingüística Sistémico Funcional y la Lingüística Textual”. LING6.

Ejemplo 6: "Este trabajo tiene como objetivo relacionar las condiciones hidrodinámicas, eventos de surgencia y estratificación de la columna de agua con los procesos de sedimentación de partículas, en la bahía de Mejillones durante un período estival". BIO10.

Al igual que en el caso anterior, se consideró la mención de "artículo", "trabajo" y de otras expresiones equivalentes, como "esta aproximación crítica", "ejercicio de escritura". En todos los casos, el artículo asumía el propósito de la investigación.

\subsubsection{EXPLICITA EL OBJETIVO DEL AUTOR}

Otro patrón identificado lo hemos categorizado como "Explicita el objetivo del autor". Bajo este patrón se formula el propósito de el o los investigadores. Este tipo de formulación corresponde al 13,3\% del total de casos. El patrón determinado es una aserción elocutiva:

$\mathrm{El}$ investigador/autor tiene (verbo presente o pasado) un objetivo (verbo en infinitivo)

Mi objetivo es + verbo en infinitivo

Este patrón se caracteriza porque el investigador/autor declara un interés, un propósito o una intención expresado en presente y asociado a un verbo en infinitivo. Este patrón también enfatiza el carácter metonímico del artículo respecto de la investigación, en la medida que la acción de investigar se confunde enunciativamente con la acción de escribir el artículo. 
Ejemplo 7: "Mi intento es poner en perspectiva dialógica escrituras que se han potenciado, secretamente, desde algún lugar imaginado, en las complicidades para la alteración de la producción cultural en nuestro continente". LIT7.

Ejemplo 8: "Lo importante para los autores, es demostrar cómo esta neutralidad se opone a la visión crítica del 11 de septiembre, aspecto que tiene una sensibilidad interna en el medio, debido a su franca oposición en los días de la Unidad Popular". HIS4.

Destaca, en estos casos, la formulación en primera persona, singular y plural. Bajo este patrón se refuerza el valor realizativo en el AIC, toda vez que el objetivo es presentado en tiempo presente y se plantea como el objetivo que se desarrollará por medio de la escritura del artículo.

Estos tres tipos de formulación explícita de objetivos, es decir, que haya sido formulado el objetivo de la investigación, del artículo o los autores, corresponden al 43,3\% de los casos.

A continuación, presentamos otras dos formas de presentación de objeticos en los AIC que consideramos menos directa que las anteriores son: "Señala lo que hace el artículo" y "Señala lo que se hace en el documento". Se incluyen en esta categoría los segmentos textuales que referían a la actividad científica como la actividad global desarrollada por el propio artículo, en el primer caso, y a la actividad global que se realiza en el artículo, en el segundo caso. Cabe precisar que no se consideraron aquí la referencia a actividades interpretadas como etapas metodológicas comúnmente descritas en los AIC.

\subsubsection{SEÑALA LO QUE HACE EL ARTÍCULO}

Cuando se señala lo que hace directamente el artículo se establece el propio artículo como agente de las acciones que se realizan y se le acompaña de un predicado verbal en presente y en tercera persona singular. Incluimos en esta clasificación las formulaciones en las que el artículo realiza acciones predicativas o realizativas. En estos casos se destaca la escritura del AIC como la actividad por medio de la cual se lleva a cabo la investigación. Se identificaron 14 menciones de este tipo 
de formulación, esto es el 26,7\%. El patrón característico lo hemos descrito como una aserción delocutiva:

El artículo + verba dicendi + actividad investigativa

$\mathrm{El}$ artículo + verbo realizativo en presente

Ejemplo 9: "El artículo describe el tratamiento periodístico del diario El Mercurio acerca del 11 de septiembre de 1973. Esta fecha, considerada un hito en la historia nacional, es objeto de un tratamiento que del tono positivo pasa a uno predominantemente neutro". HIS4.

Ejemplo 10: "Este trabajo estudia los procesos de canonización en la literatura chilena y sus relaciones con las identidades socioculturales, desde la generación romántica de 1852 (ó 1842) hasta la última década del siglo XX, delimitando sus autores, textos y metatextos canónicos”. LIT5.

En este tipo de formulación se consideró la mención de "artículo", "trabajo" u otras equivalentes como "esta revisión" o, incluso, "esta ponencia".

\subsubsection{SEÑALA LO QUE SE HACE EN EL DOCUMENTO}

Esta categoría la hemos identificado como "Señala lo que se hace en el documento". En ella, se pretende agrupar los casos en los que se propone al documento escrito como el momento y lugar donde se hace la investigación. Esta formulación se caracteriza por el uso de la preposición "en", la se manifestó en un 30\% de los segmentos textuales identificados con la función de plantear los objetivos. Si bien la formulación de los objetivos en estos casos no es explícita, se interpreta la actividad descrita como la actividad global que se reporta en el artículo. El patrón podría ser descrito como una aserción delocutiva: 
En el artículo se hace (tercera persona singular) con el propósito de + verbo en infinitivo

En el artículo + primera persona + actividades investigativas.

Ejemplo 11: "En el presente trabajo se realiza un análisis de endemicidad (Szumik et al., 2002; Szumik \& Goloboff, 2004) sobre la base de datos de distribución de 426 especies/subespecies de carábidos (Coleóptera: Carabidae), utilizando diferentes escalas de análisis (tamaños de celdas) con el fin de identificar áreas de endemismo en América del Sur austral, y de observar los efectos producidos por la variación del tamaño de celda empleado en la identificación de estos patrones". BIO4.

Ejemplo 12: "En este artículo se presenta el resultado del análisis de las propiedades morfológicas y semánticas del sufijo -ero en creaciones neológicas por sufijación en las variedades del español de Argentina, Chile y Uruguay, como una manera de verificar su vitalidad". LING10.

Ejemplo 13: "En este artículo pasamos revista a la historia de la política lingüística en los Estados Unidos de América y analizamos críticamente las diferentes posibilidades educativas que se ofrecen a los estudiantes que hablan una lengua extranjera en este país”. LING8.

En síntesis, podemos señalar que se identificaron 5 maneras de referirse a los objetivos en los AIC. A continuación, se ofrece una tabla que sintetiza los tipos de formulación de objetivos con el patrón correspondiente y los porcentajes de aparición.

Destacamos aquí que solo el $43 \%$ de las veces en que fueron formulados los objetivos de investigación se hizo de manera explícita (patrones A, B y C), mientras que el 57\% de las veces se presentaron actividades de investigación que correspondían a los objetivos de investigación (patrones D y E). 
Tabla 3: Tipo de formulación y patrón que lo caracteriza

\begin{tabular}{|c|c|c|}
\hline Tipo & Patrón & Porcentaje \\
\hline $\begin{array}{l}\text { Explicita el objetivo de la } \\
\text { investigación }\end{array}$ & $\begin{array}{l}\text {-El objetivo de esta investigación }+ \text { verbo ser en } \\
\text { pasado }+ \text { verbo en infinitivo. }\end{array}$ & $18 \%$ \\
\hline $\begin{array}{l}\text { Explicita el objetivo del } \\
\text { artículo }\end{array}$ & $\begin{array}{l}\text {-El artículo tiene (verbo en presente) por objetivo }+ \\
\text { verbo en infinitivo. } \\
\text {-El objetivo de este artículo es (verbo en presente) }+ \\
\text { verbo en infinitivo. }\end{array}$ & $12 \%$ \\
\hline $\begin{array}{l}\text { Explicita el objetivo del } \\
\text { autor }\end{array}$ & $\begin{array}{l}\text {-El investigador/autor tiene (verbo presente o } \\
\text { pasado) un objetivo (verbo en infinitivo) } \\
\text {-Mi objetivo es + verbo en infinitivo. } \\
\end{array}$ & $13 \%$ \\
\hline $\begin{array}{l}\text { Señala lo que hace el } \\
\text { artículo }\end{array}$ & $\begin{array}{l}\text {-El artículo }+ \text { verba dicenci }+ \text { actividad investigativa. } \\
\text {-El artículo }+ \text { verbo realizativo en infinitivo. }\end{array}$ & $27 \%$ \\
\hline $\begin{array}{l}\text { Señala lo que se hace en el } \\
\text { documento }\end{array}$ & $\begin{array}{l}\text {-En el artículo se hace (tercera persona singular) con } \\
\text { el propósito de + verbo en infinitivo } \\
\text {-En el artículo }+ \text { primera persona }+ \text { actividades } \\
\text { investigativas. }\end{array}$ & $30 \%$ \\
\hline
\end{tabular}

En relación con la formulación de objetivos de acuerdo con cada una de las disciplinas estudiadas, en la Tabla 4 encontramos lo siguiente:

Tabla 4: Distribución de tipo de patrón de formulación por disciplina

\begin{tabular}{l|l|l|l|l}
\hline Patrón de formulación & BIO & HIST & LING & LIT \\
\hline Explicita el objetivo de la investigación & $46 \%$ & $6 \%$ & $6 \%$ & $20 \%$ \\
\hline Explicita el objetivo del artículo & $15 \%$ & $6 \%$ & $19 \%$ & $7 \%$ \\
\hline Explicita el objetivo del autor & $0 \%$ & $19 \%$ & $13 \%$ & $20 \%$ \\
\hline Señala lo que hace el artículo & $15 \%$ & $31 \%$ & $13 \%$ & $47 \%$ \\
\hline Señala lo que se hace en el documento & $23 \%$ & $38 \%$ & $50 \%$ & $7 \%$ \\
\hline Totales & $100 \%$ & $100 \%$ & $100 \%$ & $100 \%$ \\
\hline
\end{tabular}

Frente al número total de menciones de los objetivos en los AIC de las distintas disciplinas, prácticamente, no se observan diferencias importantes: Biología 13, Historia 16, Lingüística 16 y Literatura 15. No obstante, como se desprende de la Tabla 4, cuando comparamos los patrones que se adoptan en los textos, se aprecian diferencias notables.

En este sentido, destaca en los AIC de Biología una predominancia de la formulación explícita de los objetivos de la investigación (patrón A, 46\%), a su vez, en los AIC de Literatura destaca por la formulación de lo que se hace en el documento (patrón D, 47\%). En los AIC de Lingüística se caracteriza porque mayoritariamente se señala lo que se hace en el documento (patrón E, 50\%) y en Historia se aprecia una tendencia utilizar las formas implícitas (patrones D y E, 31\% y $38 \%$ respectivamente).

ARIAS - La formulación de los objetivos en artículos de investigación científica... 
El único patrón que obtuvo frecuencia cero en algunas de las disciplinas fue el patrón C en los AIC de Biología, esto significa, que los AIC de Biología nunca presentan los objetivos como las intenciones o intereses de los investigadores.

\subsection{EL APARTADO DONDE SE FORMULAN LOS OBJETIVOS EN LOS AIC}

De acuerdo a la caracterización que hemos hecho del AIC, se esperaba que los objetivos aparecieran en el resumen y en la introducción. Sin embargo, buscamos la presencia de los objetivos en todos los apartados del AIC.

\subsubsection{OBJETIVOS EN LOS APARTADOS RESUMEN, INTRODUCCIÓN Y OTRO.}

Se consideró la aparición de uno o más segmentos textuales que mencionaran los objetivos en cualquiera de las formas de presentación descritas anteriormente. Como podemos apreciar en la Tabla 5, de los 40 resúmenes analizados, solo $77,5 \%$ incluía objetivos en cualquiera de las 5 formas de presentación que hemos estudiado. Mientras que de las 40 introducciones analizadas solo el $47 \%$ hacía referencias a los objetivos. Además, vale destacar que de todos los artículos analizados solo 3 presentaron objetivos en otro apartado distinto del Resumen o la Introducción, lo que equivale al 7,5\%.

Tabla 5: Apartado donde se reportan objetivos en los AIC

\begin{tabular}{l|r|r}
\cline { 2 - 3 } & Objetivos & $=40$ \\
\hline Resumen & 31 & $77,5 \%$ \\
\hline Introducción & 19 & $47,5 \%$ \\
\hline Otro & 3 & $7,5 \%$ \\
\hline
\end{tabular}

Igualmente, se analizaron todas las posibilidades de cómo podían presentarse los objetivos en los AIC. Se identificaron las siguiente posibilidades: no presentan objetivos (2 artículos), presentaron objetivos solo en el Resumen (15 artículos), solo en la Introducción (7 artículos). 
También se consideraron sus posibles combinaciones: aparecen simultáneamente en el Resumen y la Introducción (13 artículos), en el Resumen y Otro (3 artículos). La combinación de que aparecieran solo en Otro, en Introducción y Otro o que aparecieran en los tres apartados no tuvo ninguna ocurrencia.

Tabla 6: Apartados del artículo donde se reportan los objetivos

\begin{tabular}{l|r|r}
\cline { 2 - 3 } & Objetivos & $\mathrm{N}=40$ \\
\hline No presenta objetivos & 2 & $5 \%$ \\
\hline Solo Resumen & 15 & $38 \%$ \\
\hline Solo Introducción & 7 & $18 \%$ \\
\hline Solo Otro & - & - \\
\hline Resumen e Introducción & 13 & $33 \%$ \\
\hline Resumen y Otro & 3 & $8 \%$ \\
\hline Introducción y Otro & - & - \\
\hline Resumen, Introducción y Otro & - & - \\
\hline Total de AIC & 40 & $100 \% 0$ \\
\hline
\end{tabular}

De la información que se presenta en la Tabla 6, llaman la atención los artículos en los que los objetivos se expresaron solo en el resumen. Esta situación se da en el 38\% de los artículos estudiados. Podemos interpretar que la inclusión de los objetivos solo en el resumen refleja una concepción predominante de este apartado como un complemento del artículo. Pareciera ser que en estos artículos no se considera que el resumen sea una síntesis del artículo. A nuestro juicio, no es adecuado que se planteen aspectos importantes de la investigación en el apartado resumen y que no vuelvan a mencionarse posteriormente en el cuerpo del artículo.

Algo similar podemos decir del 18\% de los artículos en los que solo se reportan los objetivos en el apartado introducción. Al respecto se puede inferir que habría una deficiencia en la elaboración del resumen si no integra información respecto de los objetivos, la que sería relevante para que el resumen cumpla con su función de sintetizar el contenido del artículo.

De la muestra estudiada, solo el 33\% presentó los objetivos tanto en el Resumen como en la Introducción. Esta combinación es la que tiene mayor sentido, puesto que, como se ha observado, cada uno de estos segmentos textuales requiere de los objetivos para cumplir su función. 


\subsubsection{APARTADOS EN LOS QUE SE FORMULAN LOS OBJETIVOS POR DISCIPLINA}

Si atendemos ahora a la formulación de objetivos de acuerdo con cada una de las disciplinas estudiadas, encontramos que destacan los AIC de Biología que privilegian levemente la Introducción por sobre el Resumen para presentar los objetivos. En las otras tres disciplinas se prefiere claramente el Resumen. Vemos que solo en los AIC de Lingüística y de Literatura también incluyen objetivos en otro apartado: en el caso de Literatura, se presentó en la primera parte del marco teórico y en Lingüística, las dos ocurrencias fueron en un apartado destinado a la metodología.

Tabla 7: Formulación de objetivos por disciplina y apartado donde se presenta

\begin{tabular}{l|r|r|r|r}
\cline { 2 - 5 } & Resumen & Introducción & Otro & Total \\
\hline Biología & $46 \%$ & $54 \%$ & $0 \%$ & 13 \\
\hline Historia & $69 \%$ & $31 \%$ & $0 \%$ & 16 \\
\hline Lingǘstica & $63 \%$ & $25 \%$ & $13 \%$ & 16 \\
\hline Literatura & $53 \%$ & $40 \%$ & $7 \%$ & 15 \\
\hline
\end{tabular}

\subsubsection{TIPO DE FORMULACIÓN Y APARTADO EN QUE APARECE}

En la Tabla 8 se muestran en cada uno de los apartados estudiados (Resumen, Introducción y Otro) la distribución en porcentajes de los cinco patrones identificados. Llama la atención que los patrones A, B y C, que dan cuenta de la formulación explícita del objetivo de la investigación, del artículo o de los investigadores, respectivamente, predominan en el apartado introducción (77\%), esto puede interpretarse como la conciencia de que la introducción debe explicar de manera directa los propósitos de la investigación. Mientras que las formulaciones que corresponden al patrón $\mathrm{D}$ y $\mathrm{E}$ se usan, preferentemente, en el resumen (80\%), es decir, en el resumen se prefiere presentar los objetivo con el patrón que enfatiza en lo que hace el artículo y lo que se hace en el documento, lo que refuerza el carácter performativo del artículo. 
Tabla 8: Tipo de formulación y apartado en que se presenta

\begin{tabular}{l|l|l|l}
\hline Patrón & Resumen & Introducción & Otro \\
\hline A. Explicita el objetivo de la investigación & $6 \%$ & $36 \%$ & $33 \%$ \\
\hline B. Explicita el objetivo del artículo & $9 \%$ & $18 \%$ & $0 \%$ \\
\hline C. Explicita el objetivo del autor & $6 \%$ & $23 \%$ & $33 \%$ \\
\hline D. Señala lo que hace el artículo & $40 \%$ & $9 \%$ & $0 \%$ \\
\hline E. Señala lo que se hace en el documento & $40 \%$ & $14 \%$ & $33 \%$ \\
\hline TOTAL & 35 & 22 & 3 \\
\hline
\end{tabular}

\subsubsection{RELACIÓN TIPO DE PATRÓN, DISCIPLINA DE ORIGEN DEL AIC Y APARTADO EN QUE SE PRESENTA}

El Gráfico 1, que se presenta a continuación, se elaboró en base a las frecuencias totales, el que muestra la comparación entre los AIC de las diferentes disciplinas, asumiendo que están representadas cada una con 10 artículos, por lo tanto, las frecuencias brutas reflejan las diferencias efectivas que hay entre las disciplinas. Para leer el gráfico se debe considerar que este compara, dentro de los datos de cada disciplina, la inclusión de objetivos en cada uno de los apartados: Resumen (R), Introducción (I) y Otro (O). Así mismo se integra los tipos de patrones: A. Explicita el objetivo de la investigación; B. Explicita el objetivo del artículo; C. Explicita el objetivo del autor; D. Señala lo que hace el artículo; E. Señala lo que se hace en el documento.

Como se puede leer en el Gráfico 1, los AIC de Biología destacan por presentar los objetivos tanto en el Resumen como en la Introducción con una marcada preferencia por plantear explícitamente los objetivos de la investigación (patrón A) en la Introducción. Además, podemos señalar que Lingüística e Historia tienen un comportamiento similar: en ambas disciplinas se prefiere el Resumen para presentar los objetivos y se hace de una manera performativa, en la medida de que se privilegia el patrón E, "Señala lo que se hace en el documento". En tanto, los AIC de Literatura destacan por el predominio del patrón $\mathrm{D}$ cuando se presentan los objetivos en el Resumen, esto es, señalar lo que hace el artículo y la declaración de los propósitos del autor cuando se hace en la Introducción, patrón B. 


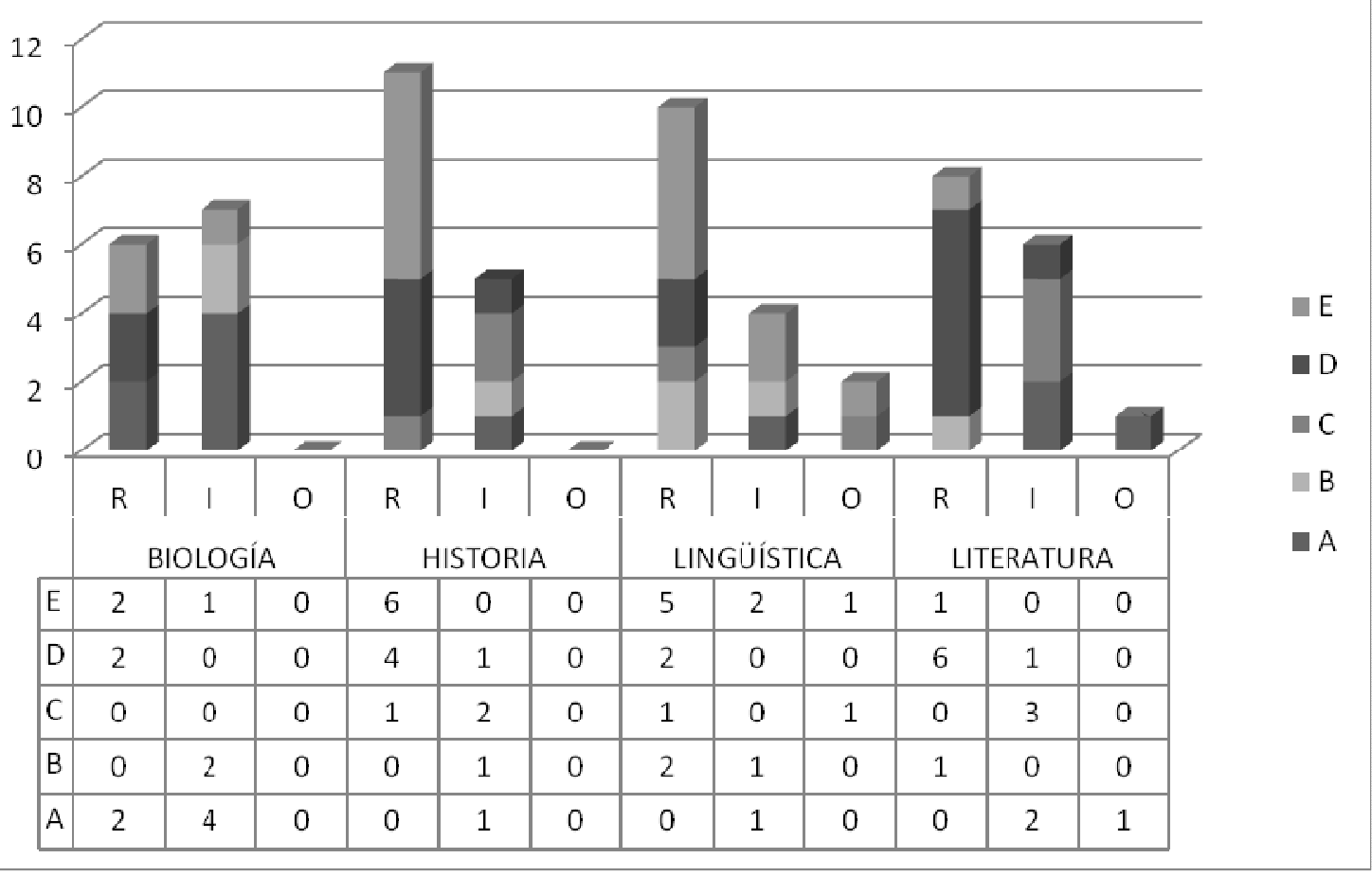

Gráfico 1: Relación tipo de formulación, disciplina y apartado en que se presenta

\section{CONCLUSIONES}

En este artículo se presentaron los resultados de un estudio que comparó la formulación de los objetivos de investigación en un corpus de textos del género AIC de cuatro disciplinas: Historia, Lingüística, Literatura y Biología. La indagación tenía por propósito determinar con datos empíricos cómo se formulan los objetivos, en qué apartados son presentados y si existían diferencias sistemáticas entre los AIC de las disciplinas estudiadas. Para ello, se recolectó un corpus de 40 artículos, diez por cada una de las disciplinas, todos provenientes de revistas indexadas en la base de datos Scielo-Chile.

A partir del análisis, hemos identificado una gran variedad de formas de presentar los objetivos en los AIC. Es así como hemos descrito cinco patrones en la presentación de los objetivos en los AIC. De estos patrones, a tres de ellos los hemos identificado como una formulación explícita por la inclusión de palabras como: objetivo, propósito, finalidad u otras. Además, se diferenció si se presentaban como el objetivo de la investigación, del artículo o de los investigadores. El patrón A destaca por ser la formulación directa y explícita de los 
objetivos de investigación. El patrón B posee un carácter metonímico en la medida que se presenta el artículo como la investigación misma. El patrón $\mathrm{C}$ presenta los objetivos de el o los investigadores como propósito, interés o intención. Las dos formas implícitas que hemos identificado, en cambio, son claramente realizativas, corresponden a una formulación indirecta. En el patrón D, es el artículo el agente de las acciones de investigación y en el patrón $\mathrm{E}$ el documento escrito se consigna como el espacio donde se realizan actividades de investigación. Estos diferentes patrones dan cuenta de las diferentes maneras de representar la relación entre las actividades de investigación y las actividades de escritura. No ha sido nuestra intención determinar la manera "correcta" de formular los objetivos en un AIC, sin embargo, estimamos que la descripción que hemos realizado de estos cinco patrones debería conducir a una reflexión acerca de la escritura académica que permitirá elegir con mayor conciencia la mejor manera de comunicar nuestra actividad científica. Sin duda, la elección de uno u otro patrón de formulación de objetivos en un AIC estará guiada por las características específicas de los objetos de estudio y los procedimientos investigativos de cada una de las disciplinas. No obstante, nos parecen preferibles las formas explícitas de formulación, dentro de las cuales, la formulación A, es decir, la formulación explícita del objetivo de investigación utilizando verbos en pretérito, es la más adecuada, especialmente, cuando se trata de enseñar a escritores en formación.

Igualmente, hemos identificado los apartados donde se presentan los objetivos. $\mathrm{Al}$ respecto, resulta sorprendente la omisión absoluta de referencias a los objetivos en dos AIC, lo que refleja una deficiencia grave de artículos publicados en revistas indexadas. Igualmente, en un $38 \%$ de los casos, el resumen es el único apartado donde se presenten los objetivos, lo que sugiere que se le asigna al resumen un valor complementario respecto del resto del artículo y no se visualiza la independencia que debería haber entre el resumen y el resto del artículo, es decir, el resumen debería sintetizar el contenido del artículo y no sustituir información que debería ir en el cuerpo del artículo.

Además, hemos observado que las formas implícitas de formulación de objetivos se presentan preferentemente en el Resumen, mientras que las formas explícitas de presentan mayoritariamente en la Introducción. Esta tendencia, nos indica que en el Resumen asume la 
función de presentar al AIC como agente de las actividades de investigación o como un lugar donde se realizan las actividades de investigación. En cambio, en la Introducción se tiende a describir la investigación en pretérito, diferenciando claramente las actividades de investigación y las actividades de escritura del AIC. Este hallazgo nos parece relevante, puesto que nos muestra una tendencia a diferenciar las funciones retóricas de ambos apartados.

Respecto a la comparación entre los AIC de las disciplinas estudiadas, encontramos marcadas diferencias. Los textos de los AIC de Biología destacan por usar tanto el Resumen como la Introducción para presentar objetivos, de manera equilibrada, no obstante ello, se prefiere la Introducción para presentar explícitamente los objetivos. Por otra parte, en Lingǘstica e Historia se opta por presentar los objetivos en el Resumen, y lo hacen de manera implícita, que como hemos dicho, resalta el carácter realizativo de la escritura del AIC. En el caso de Literatura, la manera más característica de presentar los objetivos es mediante el patrón E, "lo que se hace en el documento", en el apartado Resumen, es decir, resalta la tendencia a presentar el objetivo como la actividad global que se realiza al escribir el artículo.

Otra conclusión que nos parece relevante es la necesidad de revisar la relación que se establece entre el apartado resumen y el artículo que presenta. Encontramos que solo el 33\% de los AIC estudiados presentó los objetivos en el Resumen y la Introducción como se esperaba. Consideramos que las omisiones de los objetivos en cualquiera de los dos apartados reflejan una incongruencia con las funciones tanto del resumen, como de la introducción. La no inclusión de los objetivos en cada uno de los apartados podría estar afectando la comunicabilidad de los hallazgos científicos. El resumen debe sintetizar esquemáticamente la organización del artículo, por lo tanto, debería presentar los objetivos que son una parte fundamental de una investigación. Igualmente, la introducción debería contener los objetivos de la investigación, de esa manera tendría independencia del resumen, para poder presentar al lector las características del documento que introduce.

Otra observación que nos parece relevante es el carácter realizativo que adquieren muchos de los AIC, lo que se puede apreciar a partir de la forma en cómo se plantean los objetivos. En primer lugar, mayoritariamente, se introducen los objetivos utilizando el tiempo 
presente, lo que da el efecto de la escritura como recreación de la investigación. En otros casos, este carácter realizativo se refuerza cuando se propone que el AIC es, retóricamente, el lugar y el momento en el cual se realiza la investigación. Como hemos visto, en un alto número de casos, el "artículo" es el agente de la acción de analizar, revisar, establecer, describir, etc. Se produce, además, lo que hemos denominado como una relación metonímica entre una parte (el artículo) y el todo (la investigación que informa).

Sin lugar a dudas, se podrían describir otros patrones de formulación de objetivos y se podría avanzar en la exploración de regularidades entre los tipos de investigación y las recurrencia en las formas de presentar los objetivos u otros aspectos clave de un AIC, sin embargo, creemos que esta investigación plantea información relevante para tomar conciencia de la organización retórica del AIC en uno de sus aspectos fundamentales, los objetivos.

Desde el punto de vista de la calidad de la comunicación científica, creemos que es fundamental avanzar en la identificación y descripción de las buenas y malas prácticas de escritura en el ámbito académico. Esto servirá para que tanto escritores, como evaluadores de artículos y editores de revistas científicas cuenten con antecedentes empíricos para mejorar los aspectos retóricos de la calidad de los AIC.

\section{REFERENCIAS}

ARIAS, F. Proyecto de investigación. Guía para su elaboración. Caracas: Epistéme, 1999.

BAZERMAN, C. Shaping written knowledge: The genre and activity of the experimental article in science. Madison: The University of Wisconsin Press, 1988.

CAIVANO, L. Guía para realizar, escribir y publicar trabajos de investigación. Buenos Aires: Arquim, 1995.

DAY, R. Cómo escribir y publicar trabajos científicos. Washington:

Organización Mundial de la Salud, 2005. 
DZUNG, P. Research article abstracts in applied linguistics and educational technology: a study of linguistic realizations of rhetorical structure and authorial stance. Discourse Studies, 10, p. 231-250, 2008.

FAKHRI, A. Rhetorical properties of Arabic research article introduction. Journal of Pragmatics, v. 36, p. 1119-1138, 2004.

FALAHATI, R. The use of hedging across different disciplines and rhetorical sections of research articles 1999. [en línea]. Disponible en: $<$ http://www.sfu.ca/gradlings/NWLC_Proceedings/falahati99-112.pdf>. Consultado en: mar. 2011.

FERRARI, L. Evaluación y modalidad en artículos de investigación provenientes de diferentes disciplinas. In: CIAPUSCIO, G.; JUNGBLUTH, K.; KAISER, D.; LOPES, D. (Eds.). Sincronía y diacronía de tradiciones discursivas en Latinoamérica. Frankfurt: Veuvert, 2006. p. 27-38.

FLÓREZ, R.; TOBÓN, A. Investigación educativa y pedagógica. Bogotá: Mc Graw Hill, 2003.

GROOKES, G. Towards a validated analysis of scientific text structure. Applied Linguistics 7, p 57- 70, 1986.

GUANIPA, M. Objetivos de investigación en las ciencias sociales. 2008. [en línea]. Disponible en: <http://Gestiopolis.com>. Consultado en mayo 2008.

HENRÍQUEZ, E.; ZEPEDA, M. Elaboración de un artículo científico de investigación. Ciencia y Enfermería X, 1, p. 17-21, 2004.

HERNÁNDEZ, R.; FERNÁNDEZ, C.; BAPTISTA, P. Metodología de la investigación. México: Mc. Graw-Hill, 2004.

HYLAND, K. Authority and invisibility: authorial identity in academic writing. Journal of Pragmatics, v. 34, n. 8, p. 1091-1112, 2002.

IBÁÑEZ, R. The disciplinary text genre as a means for accessing disciplinary knowledge: A study from genre analysis perspective. In: PARODI G. (Ed.) Academic and professional discourse genres in Spanish. Amsterdam: John Benjamins, 2010. p. 189-112.

MARTÍN, P. A genre analysis of English and Spanish research paper abstracts in experimental social sciences. English for Specific Purposes, n. 22, p. 25-43, 2003. 
MARTINSSON, A. Guía para la redacción de artículos científicos destinados a la publicación. 2. ed. París: Unesco - Programa General de Información y Unisist, 1983.

MORALES, O. Aproximación discursiva al artículo de investigación (AI) odontológico hispanoamericano: implicaciones para la enseñanza del discurso académico. 2008. [en línea]. Disponible en:

<http://www.saber.ula.ve/bitstream/123456789/25963/1/aproximaci\%CC3\%B 3n_discursiva.pdf $>$. Consultado em: ene. 2011).

MORALES, O.; GONZÁLEZ, C.; TONA, J. Análisis discursivo de artículos de revisión odontológicos publicados en revistas iberoamericanas entre $1995 \mathrm{Y}$ 2005: estudio exploratorio. In: PÉREZ-LLANTADA, M.; ALASTRUÉ, R.; NEUMANN, C. (Eds.). Actas del V Congreso Internacional AELFE. Zaragoza: Prensa Universitaria de Zaragoza, 2006. p. 86-92.

MOYANO, E. Comunicar ciencia. Buenos Aires: Secretaría de Investigaciones. Universidad Nacional de Lomas de Zamora, 2000.

OZTURK, I. The textual organisation of research article introductions in applied linguistics: Variability within a single discipline. English for Specific Purposes, n. 26, p. 25-38, 2007.

PARODI G. El discurso especializado escrito en el ámbito universitario y profesional: Constitución de un corpus de estudio. Revista Signos, v. 40, n. 63, p. 147-178, 2007.

The Textbook genre and its rhetorical organization in four scientific disciplines: Between abstraction and concreteness. In: PARODI G. (Ed.) Academic and professional discourse genres in Spanish. Amsterdam: John Benjamins, 2010. p. 171-188.

RENKEMA, J. Introducción a los estudios sobre el discurso. Barcelona: Gedisa, 1999.

RIMROTT, A. The discourse structure of research articles abstracts - A rhetorical structure theory (RST) analysis. In: CARTER, N.; HADIC, L.; RIMROTT, A.; STOROSHENKO, D. (Eds.). Proceedings of the 22 ${ }^{\text {nd }}$ NorthWest Linguistics Conference (NWLC) at Simon Fraser University. Canada: Linguistics Graduate Student Association, 2007. p. 207-220.

SABAJ, O. Seminario taller para la redacción de artículos de investigación. 2007. [en prensa]. Disponible en: <http://omarsabaj.files.wordpress.com/2009/08/straidefinitivo.pdf>. Consultado en mar. 2011. 
SABAJ, O.; MATSUDA, K.; FUENTES, M. Un modelo para la homogenización de las clases textuales de la Biblioteca Scielo Chile: La variabilidad del Artículo de Investigación a través de las disciplinas. Revista Información Tecnológica, v. 21, n. 6, p. 133-148, 2010.

SABAJ, O.; TORO, P.; FUENTES, M. Un modelo de movidas retóricas para el análisis de artículos de investigación en español. Revista Onomazein (en prensa), 2011.

SWALES, J. Genre analysis. English in academic and research settings. Cambridge: Cambridge University Press, 1990.

Research genres. Explorations and applications. Cambridge: Cambridge University Press, 2004.

VALDERRAMA, J. Principales aspectos sobre la preparación de un artículo para ser publicado en una revista internacional de corriente principal.

Información Tecnológica, v. 16, n. 2, p. 3-14, 2005.

VENEGAS, R. Las relaciones léxico-semánticas en artículos de investigación científica: Una aproximación desde el análisis semántico latente. Tesis doctoral, Pontificia Universidad Católica de Valparaíso, Chile, 2005.

Recebido em: 24/05/11. Aprovado em: 11/09/11.

Title: Formulation of objectives of research articles in four disciplines: History, Linguistics, Literature and Biology

Author: Cristian González. Arias

Abstract: The analysis of the structural and functional properties of scientific activity textual products aids understanding the role of language in the construction and diffusion of scientific knowledge. Thus, the present article is based on a study of the formulation of objectives of research articles (RA) in four disciplines: History, Linguistics, Literature, and Biology. The goal was to identify patterns in the formulation of objectives, the sections where they are presented, and potential differences among the disciplines. 40 articles were analyzed, ten from each discipline. The results show five patterns in the formulation of objectives, which are presented indistinctively within the abstract and the introduction. Nevertheless, some interesting differences have been found among the $\mathrm{R} A$ of the disciplines studied. Likewise, some ambiguity is found between the presentation of the research objective and the article objective, stressing the metonymic and performative character of the $R A$.

Keywords: Specialized discourse. Research article. Research objectives. 
Titulo: A formulação dos objetivos de artigos de pesquisa em quatro disciplinas: História, Linguistica, Literatura e Biologia

Autor: Cristian González. Arias

Resumo: Analisar propriedades estruturais e funcionais dos produtos textuais da atividade cientifica ajuda a compreender o papel da linguagem na construção e na divulgação do conhecimento. Este artigo relata um estudo sobre a formulação de objetivos de artigos de investigação cientifica (AIC) em quatro disciplinas: História, Linguística, Literatura e Biologia. O objetivo maior deste estudo foi identificar padrões de formulação dos objetivos, das seções onde são apresentados e verificar se há diferenças entre as disciplinas estudadas. Um corpus constituido de 40 artigos foi analisado, distribuidos igualmente para cada uma das disciplinas estudadas. Obteve-se como resultado a identificação de cinco padrões na formulação de objetivos, que são apresentados, preferivelmente, no resumo e na introdução. Não obstante, trata-se, igualmente, das diferenças existentes entre o AIC das quatro disciplinas. Igualmente, há certas ambiguidades entre apresentar o objetivo da investigação e o objetivo do artigo, o que ressalta o caráter metonímico e performativo do $A I C$.

Palavras-chave: Discurso especializado. Artigo de pesquisa. Objetivos da investigação cientifica.

ARIAS - La formulación de los objetivos en artículos de investigación científica... 